\title{
Delayed Anaphylactic Shock to Intravenous Cefotetan in a Pregnant Woman
}

\author{
- A Case Report- \\ Ah-Reum Cho, M.D. ${ }^{*}{ }^{\ddagger}$, Hyeon-Jeong Lee, M.D., Ph.D. ${ }^{*}{ }^{\ddagger}$, \\ Hye-Kyung Park, M.D., Ph.D. ${ }^{\dagger}{ }^{\dagger}$ and Young-Jae Oh, M.D.* \\ *Department of Anesthesia and Pain Medicine, School of Medicine, Pusan National University, ${ }^{\dagger}$ Department of Internal Medicine, \\ School of Medicine, Pusan National University, ${ }^{\dagger}$ Medical Research Institute, Pusan National University, Busan, Korea
}

\begin{abstract}
Anaphylactic reactions to agents administered intravenously usually occur within minutes. We present an unusual case of a delayed onset anaphylactic shock to intravenous cefotetan in a pregnant woman who underwent an epidural cesarean section. She sustained hypotension, tachycardia, bronchospasm, and rash $90 \mathrm{~min}$ after administering intravenous cefotetan. The possibilities of high epidural blocks or amnionic fluid embolisms were excluded by the height of sensory blocks or different presenting symptoms and signs, respectively. Allergic skin tests for exposed materials were performed 6 weeks after discharge and no immediate reactions occurred. However, delayed systemic allergic reactions, such as urticaria, rash, and edema on her face, neck, back, and abdomen, occurred $3 \mathrm{~h}$ after skin test to cefotetan.
\end{abstract}

Key Words: anaphylaxis, cefotetan, skin test.

Diagnostic procedures in allergy can be classified into the patient's history, in vivo skin testing, in vitro laboratory tests and provocation tests.[1] Although skin testing is not sufficient to make diagnosis, the allergy skin testing is an important element in the evaluation of drug hypersensitivity, especially in $\beta$-lactam drug allergy. Skin testing is the most rapid, sensitive, and cost effective testing modality for the detection of IgE-mediated disease. The positive skin test is a transient "weal and flare" reaction, which represents the immediate phase of the allergic reaction. Additionally, late phase reaction also may develop in some individuals, which consist of deep tissue swelling, warmth, pruritus, and erythema beginning $1-2$ hours after testing.[2] Up to date, despite the attempts to elucidate immunologic mechanisms, the reliable diagnosis of allergic reaction is particularly difficult and anaphylaxis still remains a

Received on February 17, 2012, Revised on March 28, 2012, Accepted on March 29, 2012

Correspondence to: Hyeon-Jeong Lee, Department of Anesthesia and Pain Medicine, School of Medicine, Pusan National University, 179 Guduck-ro, Busan 602-739, Korea

Tel: 051-240-7399, Fax: 051-242-7466

E-mail: 1hjksk@ pusan.ac.kr

This work was supported by Pusan National University Research Grant 2010. clinical diagnosis.

Cefotetan disodium is a broad-spectrum second-generation cephalosporin commonly used as prophylaxis in abdominal and pelvic surgery. Although short-term prophylactic antibiotic regimens appear to carry minimal risks, such therapy has been associated with several adverse reactions, including anaphylactic shock.[3] Anaphylactic reaction during anesthesia usually occurs within minutes after anesthetic induction and is primarily linked to agents administered intravenously.[4] Although a case of delayed type skin rash[3] and a case of delayed intradermal-test positivity[5] has been reported, delayed anaphylactic shock related to the intravenous drugs has rarely been reported, especially in the anesthetic settings.

We present a case of a delayed anaphylactic shock to cefotetan in a pregnant woman who underwent an epidural cesarean section.

\section{CASE REPORT}

A 32-year-old pregnant woman (G1P0A0, weight $65 \mathrm{~kg}$, height $156 \mathrm{~cm}$ ) was admitted to Pusan National University Yangsan hospital at 39 weeks' gestation for induction of labor. 
Her past medical history and antenatal care records were unremarkable. She had worked as a laboratory assistant in the animal anatomy lab for 10 years. She denied any previous history of allergic reaction to food, drug, and latex. After negative skin test of cefotetan, the patient was transferred to the operating room. Emergency cesarean section under epidural block was planned due to cephalopelvic disproportion. She was brought to the operating room immediately and routine monitoring devices including electrocardiography (ECG), pulse oximetry, and blood pressure (BP) cuff were placed. Her vital signs revealed stable, which were BP: 140/90 $\mathrm{mmHg}$, heart rate (HR): 90 beats/min, and oxygen saturation $\left(\mathrm{SpO}_{2}\right)$ : $99 \%$. The epidural catheter with an 18-gage Touhy needle was inserted using a midline approach at the L3-L4 interspace. After negative aspiration of blood or cerebrospinal fluid, $15 \mathrm{ml}$ of ropivacaine (Naropine ${ }^{\circledR}$, AstraZeneca, UK) $0.75 \%$ and $50 \mu \mathrm{g}$ of fentanyl citrate (Fentanyl citrate, Myung Moon Pharm, Korea) was administered epidurally after hydration of $500 \mathrm{ml}$ of lactated Ringer's solution. A fourth thoracic (T4) dermatome level of sensory blockade was achieved without hemodynamic derangement. The cesarean section was begun and 2,900 $\mathrm{g}$ male baby was delivered with Apgar scores of 8 and 9 at 1 and 5 min, respectively. After delivery, we injected cefotetan (Cefotetan, Kukje Pharm, Korea) and duratocin Carbetocin, Ferring Pharm, Korea). After spreading guardix (Guardix-sol, Han Mi Pharm, Korea) to the abdominal cavity, the peritoneum was closed. Ninety min after delivery, the patient complained urticaria on both hands and mild dyspnea. The height of block was checked as T6. The itching was extended to whole body and severe air hunger occurred. She became extremely anxious and agitated; oxygen saturation was dropped to $89 \%$. We injected propofol $80 \mathrm{mg}$ and rocuronium $50 \mathrm{mg}$ intravenously. Although successful tracheal intubation was performed and ventilated with $100 \% \mathrm{O}_{2}$, the auscultation of the lungs and capnography revealed severely diminished bilateral breath sounds and almost zero end tidal carbon dioxide concentration. The high peak airway pressures above $40 \mathrm{~cm} \mathrm{H}_{2} \mathrm{O}$ was detected even at low tidal volumes of $50-100 \mathrm{ml}$. Severe bronchospasm was suspected. Her vital signs revealed that circulatory shock state (BP: 50/30 mmHg) with tachycardia (HR: 150 beats/min) without ST segment or $\mathrm{T}$ wave changes. Furthermore the pulse oximetry measurement of oxygen saturation was checked as $60 \%$ even after tracheal intubation and mechanical ventilation. Arterial cannulation in the left radial artery and central venous catheterization in the right internal jugular vein were performed. Arterial blood gas analysis (ABGA) demonstrated severe hypoxemia: $\mathrm{pH} 7.22, \mathrm{PaCO}_{2} 50.7 \mathrm{mmHg}$, $\mathrm{PaO}_{2} 41 \mathrm{mmHg}, \mathrm{SaO}_{2}$ 66\%. Epinephrine 100 ug was given intravenously and started infusion and intravenous fluids were opened to gravity. Then, the blood pressure was restored at $100 / 60 \mathrm{mmHg}$ and bronchospasm was reversed. Forty five min after the event, the patient was transferred to the surgical intensive care unit. Two liters of lactated Ringer's solution and $500 \mathrm{ml}$ of Hextend were given to the patient. Ipratropium inhalation solution was administered by a nebulizer. Chest $\mathrm{x}$-ray showed clear lung field and mechanical ventilation was continued with synchronized mandatory ventilation (SIMV) mode with $\mathrm{FiO}_{2} \quad 0.5$ and positive end-expiratory pressure of $5 \mathrm{~cm}$ $\mathrm{H}_{2} \mathrm{O}$. ABGA was improved: $\mathrm{pH}$ 7.326, $\mathrm{PaCO}_{2} 32.1 \mathrm{mmHg}$, $\mathrm{PaO}_{2} 216.4 \mathrm{mmHg}, \mathrm{SaO}_{2}$ 99.7\%. We suspected anaphylactic shock or amniotic fluid embolism (AFE). Dexamethasone $5 \mathrm{mg}$ and chloropheniramine $4 \mathrm{mg}$ were given intravenously for possibility of anaphylactic shock. DIC laboratory tests were performed to rule out amniotic fluid embolism and they were all negative (prothrombin time $11.1 \mathrm{~s}$, activated partial thromboplastin time $36.1 \mathrm{~s}$, platelet count $205,000 / \mu 1$, fibrinogen 316.4 $\mathrm{mg} / \mathrm{dl}$, and D-dimer $0.31 \mu \mathrm{g} / \mathrm{ml}$ ). Three hours after the event, the vital signs became stable without inotropics and vasopressors. She was extubated and transferred to general ward $13 \mathrm{~h}$ after the event and discharged after a week without other

Table 1. Results of Allergologic Test

\begin{tabular}{lcccccc}
\hline \hline Drugs & Patch test & Prick test & \multicolumn{3}{c}{ Intradermal test } \\
\hline Latex & Neg & & $1: 1000$ & $1: 100$ & $1: 10$ & Undiluted \\
Ropivacaine & & Neg & Neg & Neg & Neg & Neg \\
Lidocaine & & Neg & Neg & Neg & Neg & Neg \\
Fentanyl & & Neg & Neg & Neg & Neg & Neg \\
Guardix & & Neg & Neg & Neg & Neg & Neg \\
Duratocin & & Neg & Neg & Neg & Neg \\
Cefotetan & & Delayed-onset anaphylactic reaction $(3 \mathrm{~h} \mathrm{later)}$ & \\
\hline
\end{tabular}

Neg: negative test result. 
complications.

Allergic skin test for exposed material to the patient was performed six weeks after discharge (Table 1). For latex, we performed patch test. The skin was first moistened, and taped a piece of glove to the patient's upper back. The glove is left on the skin and reading was made 15 min later. The skin test for each drug was performed at four days interval. The test procedure consisted of skin prick test followed by intradermal test on the back, inspecting after $20 \mathrm{~min}$ for a wheal and flare. In the intradermal test, the first test solution was used $1: 1000$ diluted solution, followed by $1: 100,1: 10$, and undiluted at $20 \mathrm{~min}$ interval. The subsequent test solution was only injected if no reaction occurred to the previous one. Prick tests were considered positive if the mean wheal diameter was larger than $3 \mathrm{~mm}$ and accompanied by erythema with a negative response to the control saline.[6] Intradermal reactions were considered positive when the diameter of the initial wheal increased by more than $3 \mathrm{~mm}[6]$ Skin prick and intradermal tests for amide local anesthetics (ropivacane and lidocaine), fentanyl, cefotetan, duratocin, and guardix elicited no immediate local or systemic reaction, and the patient was allowed to go home with a supply of chloropheniramine $4 \mathrm{mg}$ tablets to be taken if she suffered a delayed response. However, the patient showed delayed hypersensitivity reaction such as urticaria, rash, and edema on her face, neck, back, and abdomen at $3 \mathrm{~h}$ after allergic skin test to cefotetan.

\section{DISCUSSION}

Anaphylactic shock is a severe form of anaphylaxis and characterized by vasodilatory shock, widespread edema, and difficulty in breathing. This case highlights severe delayed onset anaphylactic shock even after intravenous administration of drug could occur in a surgical patient.

During the perioperative period, IgE-mediated anaphylaxis commonly occurred by neuromuscular blocking agents and antibiotics, including penicillin and cephalosporins.[7] Drug hypersensitivity reactions are commonly classified as immediate or nonimmediate depending on their clinical presentation.[8] $\beta$ lactams are reported to induce both reactions.[9] Immediate reactions are usually induced by an IgE-mediated mechanism and occur within the first hour following the last drug administration.[10] These reactions usually appear as urticaria, angioedema, rhinitis, bronchospasm or anaphylaxis. Nonimmediate reactions may occur at any time from 1 to $48 \mathrm{~h}$ after the last drug administration and are often induced by a delayed T-cell- dependent type of allergic reaction.[11] In immediate $\beta$-lactam drug allergy an IgE-mediated reaction can be demonstrated by a positive skin prick and/or intradermal test after 20 min.[10] On the other hand, non-immediate reactions to $\beta$-lactams manifesting by cutaneous symptoms occurring more than one hour after last drug intake, are often T-cell mediated and a positive patch test and/or a late-reading intradermal test is found after several hours or days.[11]

European Academy of Allergy and Immunology (EAACI) has recommended skin tests and quantification of $\mathrm{IgE}$. Provocation tests with $\beta$-lactam antibiotics should be restricted to those patients with a suggestive history and negative $\operatorname{IgE}$ and skin test investigations.[6] In the aspect of $\beta$-lactam drug hypersensitivity, firm diagnosis was determined using immediatereading skin prick $(10.0 \%)$ and intradermal tests $(38.1 \%)$, latereading skin tests $(19.1 \%)$ or provocation tests (32.9\%).[12] In other words, only $48.1 \%$ of patients could be diagnosed as $\beta$ lactam drug allergy by routine skin tests before the operation. In order to reduce the risk for false-negative results, the diagnostic approach of anesthetic anaphylaxis is best postponed until 4-6 weeks after the acute event, because of refractoriness of the effector cell or temporarily depletion of specific IgE (sIgE) antibodies.[13] French Society for Anaesthesia and Intensive Care represented a guideline of skin test for cephalosporins and they recommended using undiluted drug for skin prick test and intradermal test.[14]

Diagnosis of anaphylactic reaction during anesthesia is not always straightforward. The etiologic diagnosis of an immediate reaction occurring intraoperatively relies on a triad including clinical, biological, and allergologic evidence.[15] The initial diagnosis of anaphylaxis is presumptive, although essential, because anaphylaxis may progress within minutes to become life-threatening. The diagnosis of intraoperative anaphylactic shock might be missed because many other pathologic conditions presented identical clinical features. Moreover, unusual clinical feature of this patient might make more difficult to diagnose intraoperative anaphylactic shock. However, we could exclude the possibility of high epidural block by height of sensory block (T6) or AFE by different presenting symptoms and signs,[16] which usually occurs with hypotension and signs of nonreassuring fetal status (100\%), pulmonary edema or respiratory symptoms (93\%), cardiac arrest (87\%), cyanosis (83\%), and coagulopathy (83\%). Although AFE has the symptoms consistent with anaphylactic shock, DIC should lead the clinician to suspect AFE. Moreover, AFE rarely occurs during cesarean section.[17] 
Even if we have high suspicion on anaphylactic shock intraoperatively, finding the allergen is a different story. It can be hampered as multiple drugs, devices, and procedures are used in the perioperative period and they can elicit heterogenous allergic and nonallergic reactions with distinct and sometimes unclear pathological mechanisms. In addition, none of the available diagnostic tests demonstrates absolute accuracy.[18] This is why diagnosis of intraoperative anaphylaxis should include multiple approaches. Although we did not perform biochemistry tests, her clinical and allergologic evidences show strong correlation of delayed onset anaphylactic shock induced by cefotetan. It could explain why preoperative skin test for cefotetan showed negative result.

In conclusion, the absence of previous history of allergy and negative skin test does not preclude the possibility of anaphylactic reaction in an anesthetic setting even at prolonged time after injection. The risk of delayed onset anaphylaxis must be borne in mind when $\beta$-lactam antibiotics planned to be administered perioperatively.

\section{REFERENCES}

1) Ring J: Pseudo-Allergic Drugs. In: Allergy: Theory \& Practice. 2nd ed. Edited by Karenblat PE, Wedner $\mathrm{HJ}$ : Philadelphia, Saunders Company. 1992, pp 243-64.

2) Brockow K, Romano A, Blanca M, Ring J, Pichler W, Demoly P: General considerations for skin test procedures in the diagnosis of drug hypersensitivity. Allergy 2002; 57: 45-51.

3) Bloomberg RJ: Cefotetan-induced anaphylaxis. Am J Obstet Gynecol 1988; 159: 125-6.

4) Kroigaard M, Garvey LH, Gillberg L, Johansson SG, Mosbech H, Florvaag E, et al: Scandinavian Clinical Practice Guidelines on the diagnosis, management and follow-up of anaphylaxis during anaesthesia. Acta Anaesthesiol Scand 2007; 51: 655-70.

5) Aihara M, Ikezawa Z: Evaluation of the skin test reactions in patients with delayed type rash induced by penicillins and cephalosporins. J Dermatol 1987; 14: 440-8.
6) Torres MJ, Blanca M, Fernandez J, Romano A, Weck A, Aberer $\mathrm{W}$, et al: Diagnosis of immediate allergic reactions to beta-lactam antibiotics. Allergy 2003; 58: 961-72.

7) Dewachter P, Mouton-Faivre C, Castells MC, Hepner DL: Anesthesia in the patient with multiple drug allergies: are all allergies the same? Curr Opin Anaesthesiol 2011; 24: 320-5.

8) Demoly P, Hillaire-Buys D: Classification and epidemiology of hypersensitivity drug reactions. Immunol Allergy Clin North Am 2004; 24: 345-56, v.

9) Baldo BA: Penicillins and cephalosporins as allergens--structural aspects of recognition and cross-reactions. Clin Exp Allergy 1999; 29: 744-9.

10) Blanca M, Cornejo-Garcia JA, Torres MJ, Mayorga C: Specificities of B cell reactions to drugs. The penicillin model. Toxicology 2005; 209: 181-4.

11) Schnyder B, Helbling A, Kappeler A, Pichler WJ: Drug-induced papulovesicular exanthema. Allergy 1998; 53: 817-8.

12) Bousquet PJ, Kvedariene V, Co-Minh HB, Martins P, Rongier M, Arnoux B, et al: Clinical presentation and time course in hypersensitivity reactions to beta-lactams. Allergy 2007; 62: 872-6.

13) Fisher M: Intradermal testing after anaphylactoid reaction to anaesthetic drugs: practical aspects of performance and interpretation. Anaesth Intensive Care 1984; 12: 115-20.

14) Mertes PM, Laxenaire MC, Lienhart A, Aberer W, Ring J, Pichler WJ, et al: Reducing the risk of anaphylaxis during anaesthesia: guidelines for clinical practice. J Investig Allergol Clin Immunol 2005; 15: 91-101.

15) Dewachter P, Mouton-Faivre C, Emala CW: Anaphylaxis and anesthesia: controversies and new insights. Anesthesiology 2009; 111: 1141-50.

16) Katircioglu K, Hasegeli L, Ibrahimhakkioglu HF, Ulusoy B, Damar H: A retrospective review of 34,109 epidural anesthetics for obstetric and gynecologic procedures at a single private hospital in Turkey. Anesth Analg 2008; 107: 1742-5.

17) Clark SL, Hankins GD, Dudley DA, Dildy GA, Porter TF: Amniotic fluid embolism: analysis of the national registry. Am J Obstet Gynecol 1995; 172: 1158-67; discussion 1167-9.

18) Ebo DG, Fisher MM, Hagendorens MM, Bridts $\mathrm{CH}$, Stevens WJ: Anaphylaxis during anaesthesia: diagnostic approach. Allergy 2007; 62: 471-87. 